\title{
REGISTRO DE CONVOLVULUS CRENATIFOLIUS RUIZ \& PAVÓN (CONVOLVULACEAE) EN MÉXICO
}

\author{
Eleazar Carranza González \\ Instituto de Ecología, A.C., Centro Regional del Bajío, Apdo. postal 386 \\ 61600 Pátzcuaro, Michoacán, México
}

\begin{abstract}
RESUMEN
Se menciona por primera vez a Convolvulus crenatifolius Ruiz \& Pavón, como parte de la flora ruderal mexicana. Esta especie es nativa de Sudamérica y desde finales del siglo XIX a la fecha se han colectado varios ejemplares en el centro de México, donde crecen como plantas adventicias en áreas perturbadas. Se proporciona además una descripción basada en los especímenes revisados y una clave para separar este taxon de $C$. bonariensis Cav. y C. equitans Benth., con los cuales está relacionado.
\end{abstract}

Palabras clave: adventicia, Convolvulus, especie nativa de Sudamérica, México.

\begin{abstract}
Convolvulus crenatifolius Ruiz \& Pavón is reported from Mexico for the first time. This species is native to South America. However, since as early as the end of the 19th century, it has been collected various times in central Mexico where it occurs in disturbed areas and is presumably adventitious. A morphological description is provided, as well as a key to separate this species from $C$. bonariensis Cav. and C. equitans Benth., two similar taxa with which $C$. crenatifolius is related.
\end{abstract}

Key words: adventitious, Convolvulus, Mexico, species native to South America.

\section{INTRODUCCIÓN}

El género Convolvulus fue propuesto por Linneo (1753), incluyendo en su publicación original 31 especies, de las cuales actualmente sólo algunas se conservan dentro de dicho taxon. Otras se acomodaron dentro de Ipomoea, Calystegia, Argyreia, Merremia, Evolvulus y Operculina.

Convolvulus se caracteriza principalmente por su estilo terminal único, con dos estigmas lineares, apenas algo aplanados y agudos en el ápice. Se distribuye 
ampliamente en regiones tropicales, subtropicales y templadas de prácticamente todo el planeta, ubicándose la mayor diversidad en el Viejo Mundo, sobre todo en Asia y contando en suma con alrededor de 250 especies. En América se encuentra una buena representación del género desde Estados Unidos hasta Argentina y Chile. La delimitación de muchas especies no es del todo clara y es notable la falta de estudios que definan la ubicación taxonómica de las mismas.

En el presente trabajo se dan a conocer varios ejemplares colectados en México que no pertenecen a las especies hasta ahora reconocidas para el país, concluyendo que se trata de C. crenatifolius Ruiz \& Pavón, elemento sudamericano que se registra por primera vez como planta adventicia en la flora mexicana.

\section{MÉTODO}

Se revisaron especímenes herborizados determinados como Convolvulus arvensis L. o $C$. equitans Benth., cuyas características mostraron notorias diferencias respecto a las de dichas especies.

Se estudiaron 31 ejemplares depositados en los herbarios ENCB, IBUG, IEB y MEXU, procedentes de seis estados del centro de la República Mexicana (Gto., Qro., Hgo., Jal., Mich. y Méx.) y del Distrito Federal.

\section{RESULTADOS Y DISCUSIÓN}

La revisión de numerosos ejemplares de Convolvulus colectados en el centro de México y determinados como $C$. arvensis $\mathrm{L}$ o $C$. equitans Benth., indica que se trata más bien de un taxon conocido de Sudamérica, descrito de Perú, cuyo nombre es $C$. crenatifolius Ruiz \& Pavón. Ésta, es una especie aparentemente originaria de Sudamérica, donde crece de manera natural en regiones húmedas de Perú, Bolivia, Brasil, Uruguay, Argentina y Chile (Sprengel, 1825; Don, 1838; Choisy, 1845; Cabrera, 1953; Macbride, 1959; O'Donell, 1959; Pontiroli, 1983). Hallier (1889) definió tres variedades para la especie (var. peruviana (=crenatifolius), var. argentinica y var. montevidensis), que no tuvieron mayor aceptación, ya que el solapamiento entre ellas es demasiado estrecho y a pesar de que un argumento para separarlas es el geográfico (O'Donell, op. cit.), no se encuentra sustento suficiente para mantenerlas como entidades distintas.

Los ejemplares revisados evidentemente no corresponden a $C$. arvensis $\mathrm{L}$., ya que difieren de dicha especie en el tamaño de los sépalos y de las flores. Los primeros son de mayor tamaño (de 6.5 a $9 \mathrm{~mm}$ de largo, mientras que en $C$. arvensis cuando mucho alcanzan $5 \mathrm{~mm}$ ) y la corola es más pequeña (de 10 a $18 \mathrm{~mm}$ de 
longitud vs. 15 a $30 \mathrm{~mm}$ en $C$. arvensis). Además existen otras discrepancias a nivel de las hojas y de la pubescencia en toda la planta.

Igualmente, bajo el nombre de C. equitans Benth. han sido consideradas algunas de las colectas de México, pero la observación cuidadosa, muestra diferencias significativas respecto a tal especie tanto en la forma de las hojas y de los sépalos, como en la superficie de la semilla y en el número de flores por inflorescencia. Por su parte los individuos mexicanos se asemejan asimismo a $C$. bonariensis Cav., originario de Sudamerica, primordialmente en el tamaño de la corola, que según O'Donell (op. cit.), en esta especie oscila entre 10 y $16 \mathrm{~mm}$ de largo; sin embargo, varios caracteres de las hojas, los sépalos y los estilos, distinguen a tal planta de C. crenatifolius (Cuadro 1).

Otro nombre que aparece en la literatura y que corresponde a vegetales con morfología similar, es Convolvulus hermannioides A. Gray, descrito de Texas y citado también del norte de México. De acuerdo con la descripción original, se trata de una planta seríceo-tomentulosa, con hojas oblongas u oblongo-lanceoladas, pedúnculos con 1 o 2 flores, sépalos ovalado-oblongos y corola de alrededor de $2.5 \mathrm{~cm}$ de largo. Tales características, unidas al análisis de numerosos ejemplares y poblaciones naturales, han llevado a varios autores a la conclusión de que $C$. hermannioides sólo es un extremo de variación de C. equitans.

Cabe señalar que los ejemplares de $C$. crenatifolius de México proceden de sitios que están ligados directamente a las actividades humanas. Todos los ejemplares revisados provienen de terrenos baldíos dentro de zonas urbanizadas, orillas de caminos, carreteras o vías férreas, lo cual muestra un evidente comportamiento ruderal. Tal circunstancia indica que, con toda probabilidad, se trata de un elemento adventicio, cuya introducción al país no debe ser tan reciente, ya que la primer colecta registrada data de hace más de 100 años (M. Bárcena 227 (MEXU), mayo de 1887). Además, el carácter ruderal de estas plantas define una diferencia significativa respecto a $C$. equitans, que si bien tiene algunas poblaciones en áreas alteradas, no propiamente es una planta de zonas en constante exposición a las actividades del hombre.

La descripción de los individuos procedentes de la República Mexicana queda de la siguiente forma:

Convolvulus crenatifolius Ruiz \& Pavón, Fl. Per. et Chil. 2: 10. 1799. tab. 118. Fig. 1.

Planta voluble o decumbente, muy ramificada; ramillas glabras a tomentosas, especialmente en las extremidades; pecíolos de $0.7-2.5(3) \mathrm{cm}$, láminas foliares elípticas, ovadas u ovado-lanceoladas de $2.5-7(9) \mathrm{cm}$ de largo, de $1-3.5(5) \mathrm{cm}$ 


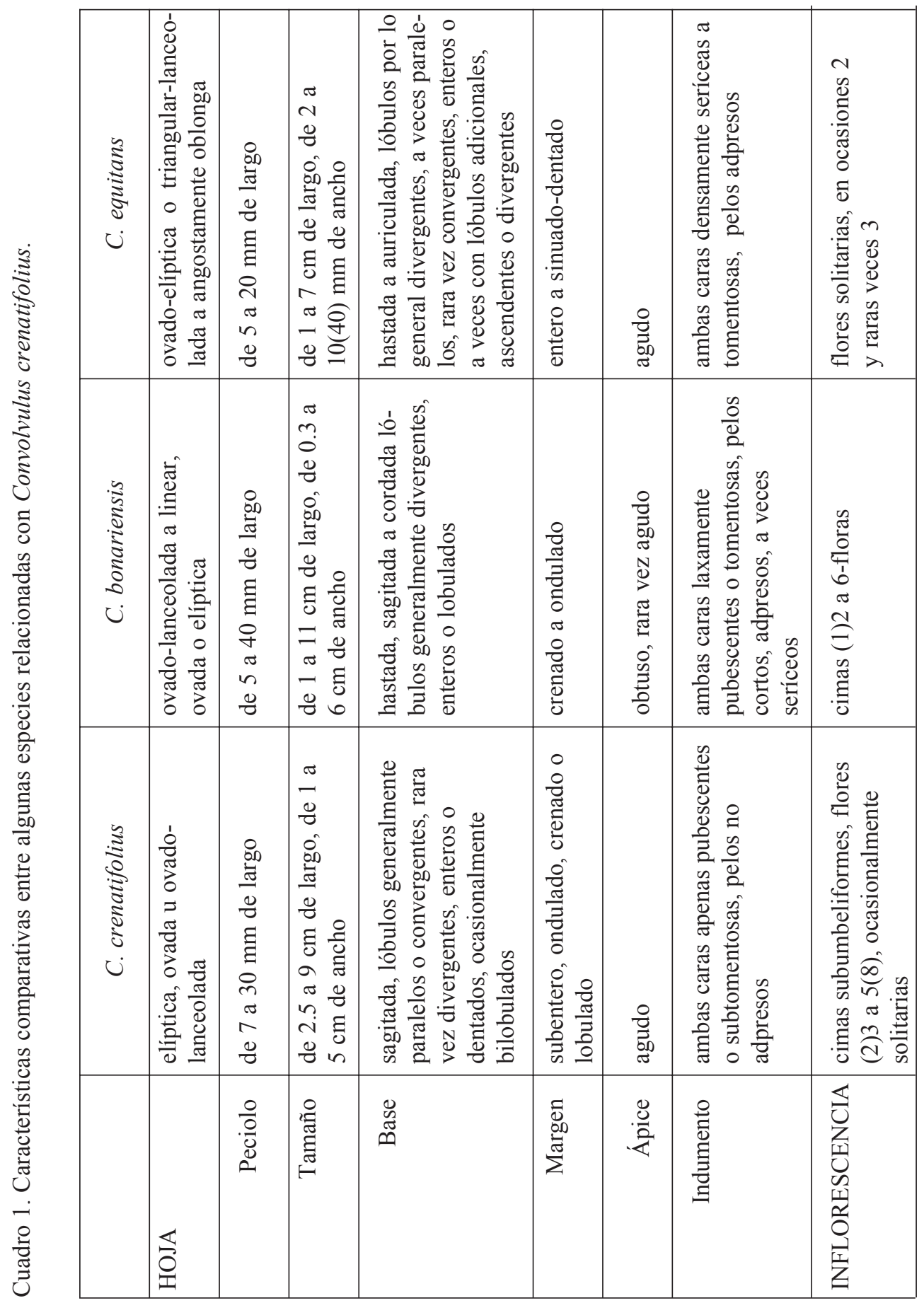


Carranza: Registro de Convolvulus crenatifolius en México

\begin{tabular}{|c|c|c|c|c|c|c|c|}
\hline 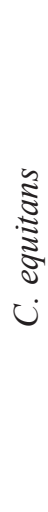 & 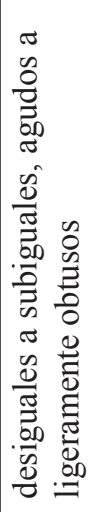 & 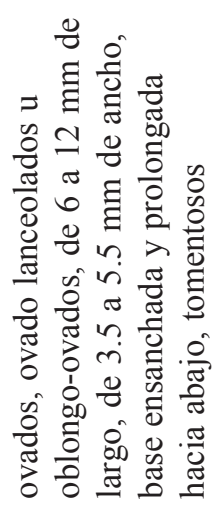 & 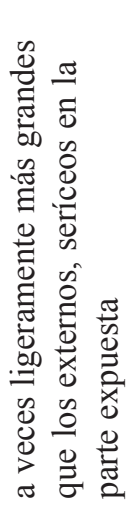 & 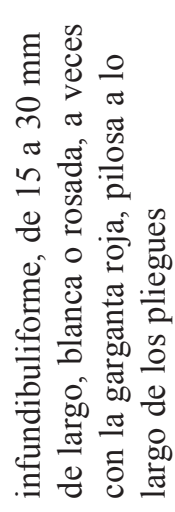 & 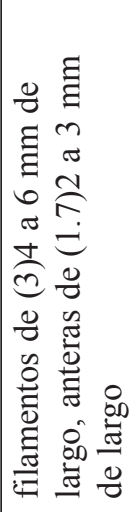 & $\begin{array}{l}0 \\
0 \\
\vdots \\
0 \\
0 \\
\Xi \\
\Xi \\
0 \\
\sigma \\
\infty \\
0 \\
0\end{array}$ & 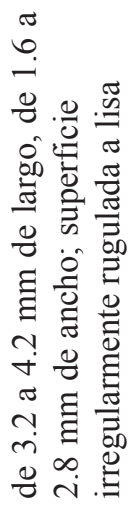 \\
\hline 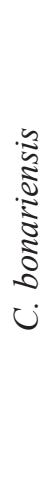 & 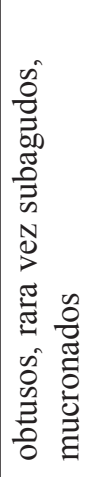 & 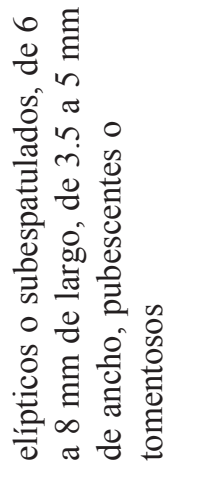 & 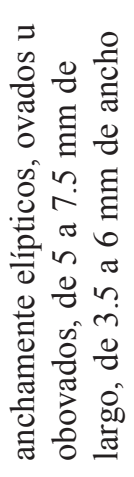 & 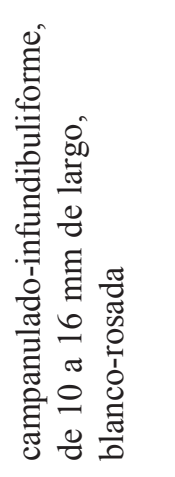 & 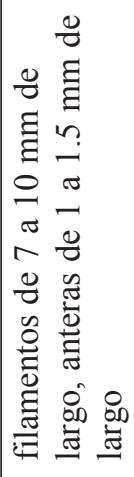 & 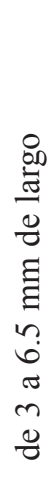 & 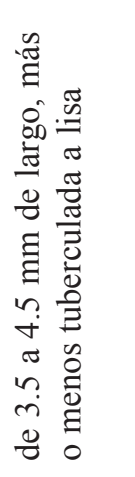 \\
\hline $\begin{array}{l}3 \\
-3 \\
0 \\
0 \\
0 \\
0 \\
0 \\
0 \\
ن\end{array}$ & 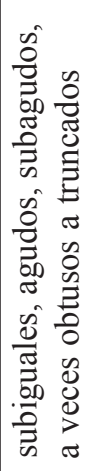 & 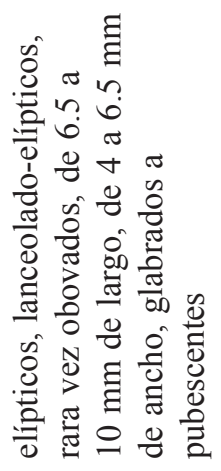 & 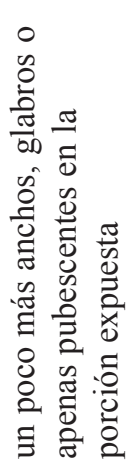 & 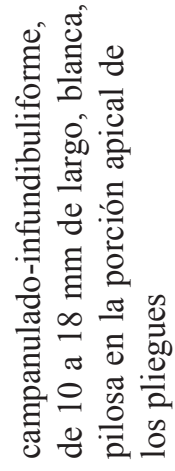 & 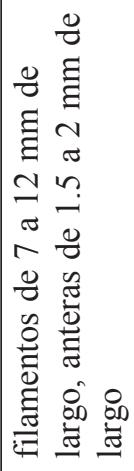 & 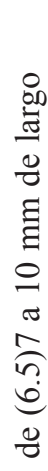 & 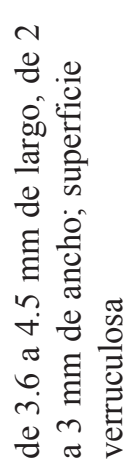 \\
\hline & 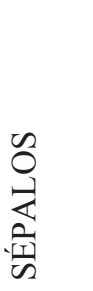 & 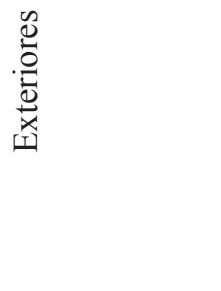 & 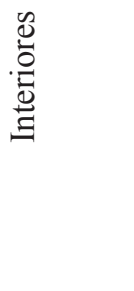 & $\begin{array}{l}0 \\
0 \\
0 \\
0\end{array}$ & 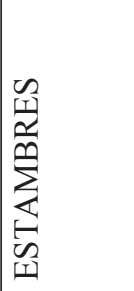 & 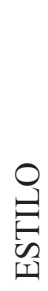 & 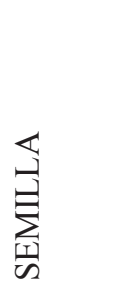 \\
\hline
\end{tabular}




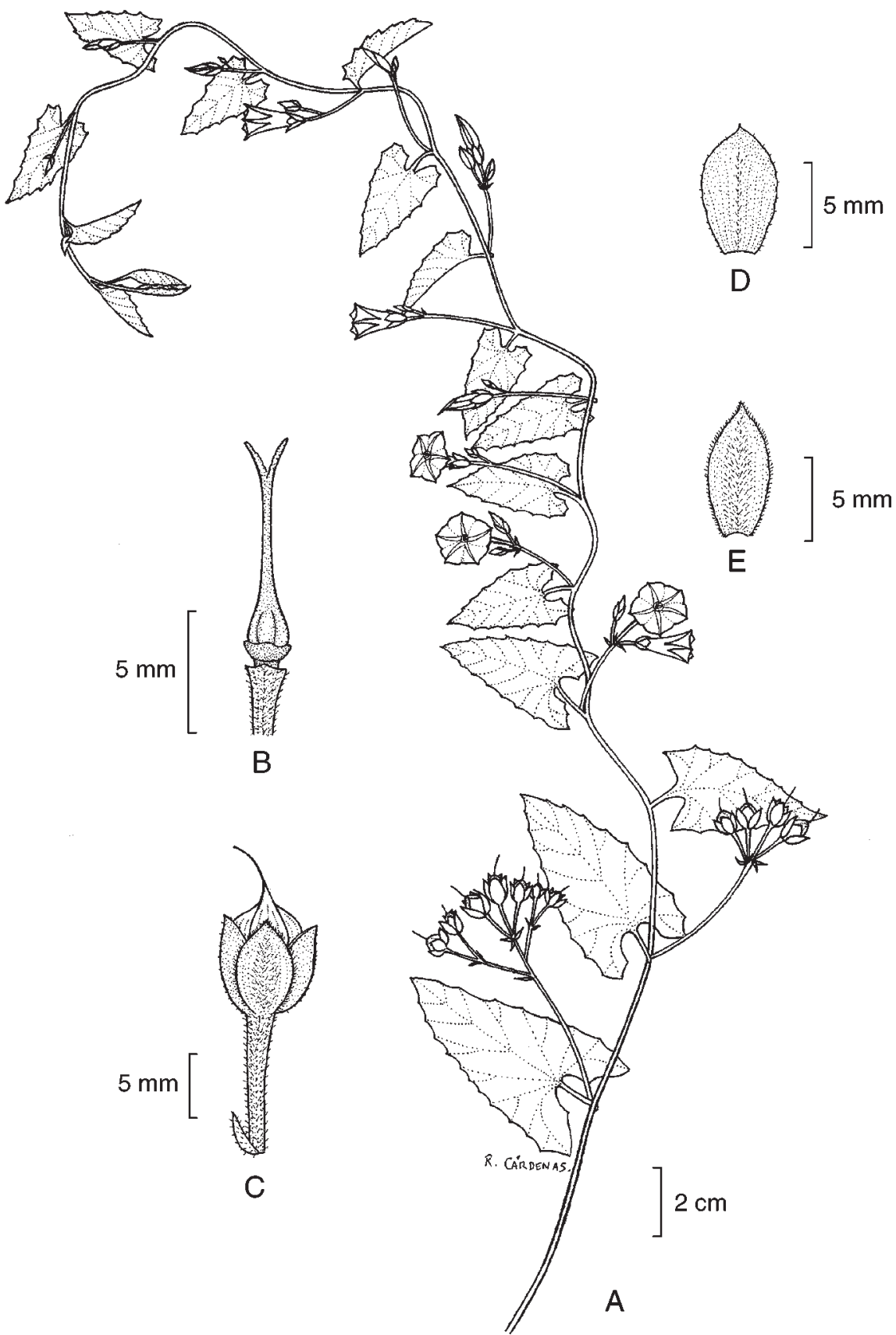

Fig. 1. Convolvulus crenatifolius Ruiz \& Pavón. A. rama con flores y frutos; B. ovario, estilo y estigmas; C. fruto; D. sépalo interno; E. sépalo externo. Ilustración de Rogelio Cárdenas. 
de ancho, subenteras o con los bordes ondulados, crenados o a veces algo lobulados, ápice agudo, base sagitada, con lóbulos generalmente paralelos o convergentes, raras veces divergentes, enteros o dentados, ocasionalmente bilobulados, apenas pubescentes a subtomentosas en ambas caras, los pelos simples, más rara vez glabras o glabrescentes; cimas subumbeliformes, de (2)3 a 5(8) flores, o éstas a veces solitarias, pedúnculos de $2-8 \mathrm{~cm}$ de largo, pubescentes, brácteas lanceoladas a lineares, de 2 - $5 \mathrm{~mm}$ de largo, pubescentes, bracteolas de 1.5 - $3 \mathrm{~mm}$ de largo, similares a las brácteas, pedicelos de $0.5-1.6 \mathrm{~cm}$ de largo, glabrescentes a pilosos; sépalos exteriores elípticos, lanceolado-elípticos o raras veces obovados, de 6.5 $9 \mathrm{~mm}$ de largo por 4 - $6 \mathrm{~mm}$ de ancho, agudos, subagudos o a veces obtusos a truncados, mucronulados, pubescentes a glabrados, los interiores un poco más anchos, glabros o apenas pubescentes en la porción expuesta; corola campanulada a más o menos infundibuliforme, de (10)12 a 16(18) $\mathrm{mm}$ de largo, blanca, pilosa en la porción apical de los pliegues, pubescencia que es más evidente en botón; estambres de 7 a $12 \mathrm{~mm}$ de largo, filamentos glabros, anteras de 1.5 a $2 \mathrm{~mm}$ de largo; ovario ovoideo, estilo de (6.5)7 a $10 \mathrm{~mm}$ de largo, glabro, estigmas de alrededor de $2 \mathrm{~mm}$ de longitud; cápsula subglobosa, glabra u ocasionalmente algo pilosa, de (5.5)6.5 a $8 \mathrm{~mm}$ de diámetro; semillas negras, de 3.5 a $4.5 \mathrm{~mm}$ de largo, de 2 a $3 \mathrm{~mm}$ de ancho, superficie algo verruculosa.

Por lo general las plantas mexicanas posen flores y frutos de menor tamaño que la mayoría de las sudamericanas. La altitud registrada en México varía de 1250 a $2400 \mathrm{~m}$. Principalmente florece de marzo a junio y fructifica de abril a agosto, aunque sobre todo en las zonas de mayor altitud, se le puede observar con flores y frutos a lo largo del año.

Material revisado: Guanajuato. Municipio de Apaseo El Alto: N de Apaseo el Alto, en zona urbana, cerca de la carretera, E. Carranza y E. Pérez 4990, 14.VII.1996 (IEB, MEXU). Municipio de Salvatierra: Salvatierra, J. Rzedowski 39598 a , 14.III.1986 (ENCB, IEB); $14 \mathrm{~km}$ al W de Salvatierra, sobre la carretera a Yuriria, J. Rzedowski 38685, 1.VII.1985 (ENCB, IEB, MEXU). Querétaro. Municipio de Landa: $3 \mathrm{~km}$ de La Vuelta, camino a Xilitla, E. Carranza 1603, 6.IV.1989 (IEB); $\pm 1.5 \mathrm{~km}$ al norte de Otates, E. González 890, 23.VIII.1989 (IEB). Municipio de Querétaro: ranchos Urquiza, E. Argüelles 352, 18.IV.1976 (MEXU); camino a Celaya, km 15 aproximadamente, E. Argüelles 429, 11.VII.1976 (MEXU). Hidalgo. Municipio de Ixmiquilpan: Ixmiquilpan, V. Fragoso 116, 7.IX.1950 (ENCB). Jalisco. Municipio de Amatitán: rancho Polioria, A. Calatayud s. n., 17.IV.1978 (IBUG). Municipio de Guadalajara: Guadalajara, M. Bárcena 227, V.1887 (MEXU); vía del tren Mariano Otero cruzamiento con Washington, sector Juárez, Guadalajara, R. Gutiérrez s. n., 17.IV.1978 (IBUG). Municipio de Tlaquepaque: presa de Toluquilla, G. J. Jara, s. n., s. f. (IBUG). Municipio de Santa 
Ana Acatlán: Santa Ana Acatlán, J. Carmona s. n., 4.V.1979 (IBUG); arroyo de la Lima cercano al poblado El Divisadero, L. M. Villarreal de Puga 8391, 28.I.1976 (IBUG). Municipio de Chapala: San Antonio, en la rivera de Chapala, J. F. Díaz s. n., 4.IV.1979 (IBUG); San Antonio Tlayacapan, S. C. Espinoza s. n., s.f. (IBUG); Chapala, H. Cárdenas s. n., 4.V.1979 (IBUG); a orillas de la laguna de Chapala, A. Covarrubias s. n., 7.IV.1979 (IBUG). Municipio de Zacoalco: El Tule, al N del Valle de Zacoalco, L. M. Villarreal de Puga 3348, 22.VI.1969 (ENCB, IBUG). Municipio de Amacueca: $8 \mathrm{~km}$ de la desviación a Tapalpa, E. Villegas y R. Ramírez 340, 9.VII.1993 (IBUG, MEXU). Municipio de Atoyac: al SE de Atoyac, R. Ornelas s. n., 16.III.1979 (IBUG). Municipio de Sayula: huerta de Citrus en las afueras NW de Sayula, D. Ramírez s. n., 2.V.1986 (IBUG). Michoacán. Municipio de Zamora: Jacona-Zamora, L. M. Villarreal de Puga 1367, 12.IV.1968 (IBUG). Municipio de Chilchota: alrededores de Chilchota, E. Carranza 5725, 4.IV.1999 (IEB). Municipio de Pátzcuaro: Pátzcuaro, jardines del Instituto de Ecología, A.C., Centro Regional del Bajío, E. Carranza 6115, 26.I.2001 (IEB). Municipio indeterminado: potrero Cuatro Esquinas, R. Pérez s. n., 10.VI.1986 (IBUG). México. Municipio de Naucalpan: Fraccionamiento Bosque de Echegaray, G. Díaz 102, 5.VIII.1969 (ENCB). Distrito Federal. Delegación G. A. Madero: colonia González Romero, Eje 3 Oriente, entre C. Gloria y Palmiro, H. Vibrans 4467, 20.VIII.1993 (IEB). Delegación Azcapotzalco: Colonia Rosario, vía del tren, atrás del campus de la UNAM, H. Vibrans 4572, 17.IX.1993 (IEB). Delegación M. Hidalgo, calle Legaria, Panteón Francés, H. Vibrans 4694, 16.I.1994 (IEB). Delegación B. Juárez, a lo largo de la vía del tren que va a observatorio hacia el sur, cerca unidad habitacional Plateros, H. Vibrans 6579, 7.V.1999 (IEB).

Clave para diferenciar C. crenatifolius de C. bonariensis y de C. equitans:

1 Sépalos subiguales, los externos ligeramente más largos que los internos; estilo de 3 a $6.5 \mathrm{~mm}$ de largo; anteras de 1 a $1.5 \mathrm{~mm}$ de longitud; hojas con el ápice obtuso, rara vez agudo, lóbulos basales divergentes; superficie de la semilla más o menos tuberculada a lisa ............................................ C. bonariensis

1 Sépalos desiguales o subiguales, pero los externos ligeramente más cortos que los internos; estilo de (6.5)7 a $16 \mathrm{~mm}$ de largo; anteras de 1.5 a $3 \mathrm{~mm}$ de longitud; hojas con el ápice agudo, a veces redondeado, lóbulos basales paralelos, convergentes o divergentes; superficie de la semilla verruculosa o rugulada a lisa.

2 Hojas cuando mucho dos veces más largas que anchas, apenas pubescentes o subtomentosas, pelos no adpresos; flores en grupos de 3 a 5(8), ocasionalmente solitarias o en pares; anteras de 1.5 a 2 mm de largo; corola 
de (10)12 a 16(18) mm de largo, blanca, nunca con la garganta rojiza; sépalos externos elípticos, lanceolado-elípticos, rara vez obovados, glabrados a pubescentes, la base nunca ensanchada ni prolongada hacia abajo; suferficie de la semilla verruculosa

C. crenatifolius

2 Hojas por lo común más tres veces más largas que anchas, densamente seríceas a tomentosas, pelos adpresos; flores solitarias, en ocasiones 2 o 3; anteras de (1.5)2 a $3 \mathrm{~mm}$ de largo; corola de 15 a $30 \mathrm{~mm}$ de largo, blanca o rosada, a veces con la garganta rojiza; sépalos externos ovados, ovadolanceolados u oblongo-ovados, tomentosos, frecuentemente con la base ensanchada y prolongada hacia abajo; superficie de la semilla irregularmente rugulada a lisa

C. equitans

\section{AGRADECIMIENTOS}

Agradezco al Dr. Jerzy Rzedowski la crítica revisión del manuscrito, así como a los curadores de los herbarios ENCB, IBUG, IEB y MEXU por las atenciones otorgadas en la revisión del material de sus acervos. Igualmente se agradece a la Dra. Heike Vibrans, quien amablemente puso a disposición material colectado en el Distrito Federal. El trabajo se llevó a cabo gracias al apoyo económico del Instituto de Ecología, A.C. (cuenta 902-07), del Consejo Nacional de Ciencia y Tecnología y de la Comisión Nacional para el Conocimiento y Uso de la Biodiversidad.

\section{LITERATURA CITADA}

Cabrera, A. L. 1953. Convolvulus. In: Manual de la flora de los alrededores de Buenos Aires.

Ed. ACME, S. A. Buenos Aires, Argentina. Vol. 5. pp. 382-383.

Choisy, J. D. 1845. Convolvulus. In: DC. Prodr. 9: 399-416.

Don, G. 1838. Convolvulaceae. In: A general history of the dichlamideous plants. London. Vol. 4. pp. 252-306..

Hallier, H. 1889. Jahrb. Hamb. Wissens. Anst. 16: 34-35.

Linneo, C. 1753. Convolvulus. In: Species plantarum. Holmiae (Estocolmo). Vol. 1. pp. 153159.

Mcbride, J. F. 1959. Convolvulus In: Flora of Peru. Field Mus. Nat. Hist. Bot. 13: 522525.

O'Donell, C. 1959. Convolvulus. In: Convolvuláceas argentinas. Lilloa 29: 262-299.

Pontiroli, A. 1983. Convolvulaceae. In: Cabrera, A. L. Flora de la Provincia de Jujuy. Colección Científica del INTA, Buenos Aires, parte VIII. pp. 177-222. 
Acta Botanica Mexicana 73: 59-68 (2005)

Sprengel, C. 1825. Convolvulus. In: Systema Vegetabilium. Goettingae. Vol. 1. pp. 590614.

Recibido en agosto de 2004. Aceptado en julio de 2005. 